\title{
Strasbourg, entre France et Allemagne. Structure urbaine et symboliques de la dualité frontalière
}

Strasbourg between France and Germany: spatial organisation and symbolics of border duality

Straßburg zwischen Frankreich und Deutschland: städtische Struktur und

Symbolik der Grenzdualität

\section{Antoine Beyer}

\section{(Q) OpenEdition}

\section{Journals}

Édition électronique

URL : http://journals.openedition.org/rge/3207

DOI : $10.4000 /$ rge.3207

ISSN : 2108-6478

\section{Éditeur}

Association des géographes de l'Est

Édition imprimée

Date de publication : 1 avril 2007

ISSN : 0035-3213

\section{Référence électronique}

Antoine Beyer, «Strasbourg, entre France et Allemagne. Structure urbaine et symboliques de la dualité frontalière ", Revue Géographique de l'Est [En ligne], vol. 47 / 2 | 2007, mis en ligne le 01 décembre 2011, consulté le 08 septembre 2020. URL : http://journals.openedition.org/rge/3207 ; DOI : https://doi.org/ $10.4000 /$ rge. 3207

Ce document a été généré automatiquement le 8 septembre 2020

Tous droits réservés 


\title{
Strasbourg, entre France et
} Allemagne. Structure urbaine et
symboliques de la dualité
frontalière

\author{
Strasbourg between France and Germany: spatial organisation and symbolics of \\ border duality \\ Straßburg zwischen Frankreich und Deutschland: städtische Struktur und \\ Symbolik der Grenzdualität
}

Antoine Beyer

\section{Introduction}

1 Comme l'Alsace, dont elle est la capitale, Strasbourg traduit spatialement des enjeux territoriaux entre la France et l'Allemagne. Les traces de ses appartenances successives sont bien lisibles dans le paysage urbain, d'autant plus que les grandes réalisations urbanistiques ont toujours constitué des manifestes de légitimation politique.

2 Aujourd'hui, le vaste projet d'urbanisation Est-Ouest, qui cherche à ouvrir Strasbourg sur le Rhin et l'Allemagne, s'inscrit bien dans cette perspective, tout en y introduisant des ruptures significatives. D'abord la reconquête des anciennes friches douanières, militaires et industrielles, marque la volonté de réinterprétation urbaine de la frontière comme trait d'union et non plus comme séparation. Elle procède de l'investissement civil du glacis défensif, jusque là mis sous la tutelle de l'Etat, qu'il ait été français ou allemand.

3 Aussi l'ambitieuse création d'un lien urbain transfrontalier cherche-t-elle à organiser l'existence d'une agglomération franco-allemande. En projetant une continuité urbaine avec sa voisine Kehl, Strasbourg souhaite à la fois incarner le rapprochement entre les deux pays, mais surtout saisir l'opportunité d'un potentiel métropolitain jusque là 
bridé par la frontière. L'ampleur et le propos du projet renouent de fait avec une histoire urbaine longue. Il offre à la ville l'occasion de se réconcilier avec un passé traversé par cette perspective binationale. Le caractère franco-allemand qu'on prête à Strasbourg est bien l'élément-clé de sa compréhension urbaine.

L'objectif de cet article est de valider cette proposition fondatrice à travers l'analyse de plusieurs éléments de la ville : la position frontalière, l'interprétation d'éléments caractéristiques de sa structure urbaine et les stratégies de développement urbain mises en œuvre. Pour ce faire, nous avons tenté de réaliser une approche systématique de la notion de dualité urbaine appliquée au contexte frontalier strasbourgeois. Dans un premier temps, le propos portera ainsi sur les conditions d'émergence et d'évolution de la dualité urbaine dans une situation frontalière. Il s'attachera ensuite à déceler l'expression de cette double appartenance dans les formes urbaines, et plus particulièrement dans l'aménagement de lieux emblématiques du pouvoir. Enfin, il montrera comment nombre de grands projets strasbourgeois actuels s'inscrivent explicitement dans le dialogue franco-allemand.

Sur le plan théorique, l'approche s'appuie sur deux grandes entrées géographiques: une réflexion sur les villes frontalières et la symbolique de l'espace urbain. Développée par Joël Kotek, la notion de villes-frontières (Kotek, 1996, Reitel 2002) fournit un cadre conceptuel précieux, qui permet de confronter le cas strasbourgeois à des trajectoires d'autres villes européennes. Notre analyse s'appuiera aussi sur les acquis théoriques et les réflexions menées actuellement en géographie sur la symbolisation des lieux, c'està-dire, pour reprendre l'expression de Jérôme Monnet «la manière dont l'organisation d'un espace et un système de valeurs s'imprègnent l'une de l'autre» (Monnet 1998, Debarbieux 1996). Dans l'interprétation symbolique de la ville que nous avons retenue (Claval 1999), nous infléchissons la problématique avancée par Vincent Calay sur la production des espaces imaginaires des villes Capitales de l'Europe, dans la mesure où selon nous, l'identité " européenne " d'une ville passe en premier par sa capacité historique de confrontation et de dialogue entre les cultures des pays de l'Union.

\section{La figure géographique de la dualité urbaine aux frontières}

\section{A. L'expression successive des trois grands types de ville aux frontières}

6 D’après la terminologie générique qu'il propose, Joël Kotek fait de Strasbourg le type même de la ville-frontière devenue ville frontalière. Pour lui, une ville-frontière est une ville disputée par deux communautés, indépendamment de sa position géographique, même si elles sont le plus souvent en position frontalière. Il signifie par là le changement fondamental de statut de la capitale alsacienne, naguère ville bipolaire, traversée par des conflits nationaux, car «contestée par deux puissances étatiques à la charnière d'ensembles ethniques ou idéologiques » (Kotek 1996). Devenue ville frontalière après 1945 , Strasbourg se définirait par sa seule position de proximité des limites nationales. Le schéma traduit alors une évolution qui a conduit à l'apaisement politique de sa situation par la disparition d'une légitimité double. Les villes-frontières ayant perdu leur statut se trouvent en fait dans des situations qui peuvent être diverses selon les modalités de leur "normalisation ». Pour rendre 
compte de l'évolution actuelle, il faut alors compléter la typologie de Joël Kotek par un troisième type de ville qui établit un nouveau mode de relation urbain à la frontière : l'agglomération transfrontalière. D'abord fait morphologique et fonctionnel, l'agglomération transfrontalière tend à revêtir aujourd'hui une dimension politique et gestionnaire. Elle s'apparente dans les faits à une intercommunalité transnationale et correspond à des formes d'organisation émergentes qui se développent et s'institutionnalisent dans un contexte européen de frontières pacifiées et de plus en plus perméables. La frontière, jusque là plutôt restrictive, voire répulsive, devient un attracteur du fait urbain.

7 Si la ville frontalière proposait une solution de normalisation dans un cadre national, l'agglomération transfrontalière cherche à construire un nouveau mode de gestion, de gouvernance, en s'appuyant sur une nouvelle identité revendiquée de la frontière qu'elle mobilise pour en tirer sa légitimité d'action. Depuis 2005, l'agglomération Strasbourg-Kehl appelée à s'approfondir et à se développer dans le cadre de l'Eurodistrict, regroupe les communes de la Communauté urbaine de Strasbourg et l'arrondissement badois voisin de l'Ortenau avec son chef-lieu offenbourg.

\section{B. La dualité structurelle des villes-frontières}

8 Dans le passage de la ville frontière à la ville frontalière, l'analyse de Joël Kotek reste toutefois par trop simplificatrice en supposant que la fin des conflits ouverts conduirait à une évidente normalité urbaine, comme si les luttes, effectives et symboliques, auxquelles se sont livrés les pouvoirs concurrents, ne laissaient aucune trace durable dans la mémoire des pierres et des hommes. Car l'histoire de ces basculements démontre que les événements ont été durablement traumatiques pour les villes comme pour leurs habitants. Ils correspondent à des changements brutaux de systèmes politiques, linguistiques, à des destructions, voire à des déplacements de population.

La disparition du caractère conflictuel de la ville-frontière peut ainsi laisser des traces profondes. La frontière naguère vive est alors souvent vécue comme une dualité héritée, traversée par une fracture devenue latente. Elle se traduit par un refoulement sélectif du passé où s'opèrent à la fois des phénomènes de dénégation et un travail continuel de justification et d'interprétation. Le conflit autrefois ouvert s'intériorise, laissant subsister un malaise diffus des populations, d'autant que le non-dit de la première génération est souvent devenu indicible pour les suivantes.

Que l'on songe ici aux villes polonaises et russes qui ont connu les marques les plus dramatiques de cette histoire, avec des déplacements massifs de populations urbaines et la destruction importante du patrimoine bâti. Le cas de Gdansk est emblématique de la complexité de cette histoire : ville désertée par sa population allemande et rebâtie à l'identique par les populations polonaises, déplacées de territoires ruraux devenus biélorusses ou ukrainiens en 1945. Les conflits et les appartenances du passé ressurgissent, allant jusqu'à constituer un puissant marqueur d'identité urbaine collective autant subi que revendiqué. Ils viennent alimenter un imaginaire spécifique, qui engage la cité dans un indispensable travail de mémoire et de compréhension du passé. C'est sur ce travail que peuvent alors se construire de nouveaux projets. L'imprégnation de la structure duale semble un trait fort des villes aux frontières. Bon nombre de villes d'Europe, peut-être même les plus « européennes » d'entre elles, ont 
connu, comme Strasbourg, cette histoire troublée par des appartenances nationales successives.

\section{La dualité frontalière, figure géographique fondatrice de Strasbourg}

11 Qu'elles soient villes-frontières, mais aussi villes frontalières ou agglomérations transfrontalières, elles se comprennent comme des villes partagées, avec toute la polysémie que peut recouvrir ce terme. La notion de partage renvoie en effet à un champ sémantique très large qui va de la partition territoriale brutale aux conflits intimes évoqués plus haut. Elle renvoie aussi à la mise en commun de fonctions et d'espaces des agglomérations transfrontalières. Cette extension sémantique fait appel à la dialectique fondatrice du concept de frontière, tout à la fois ligne de séparation (fonction de coupure) et espace de contact (fonction d'articulation) (Groupe frontière 2005). Et c'est bien à la lumière de cette tension frontalière, de la gestion de cette coupure qu'il faut analyser les trajectoires de ces villes.

12 Dans le cas de Strasbourg, cette tension est d'autant plus structurante qu'elle a été activée à plusieurs reprises dans l'histoire de la ville. Ainsi, et c'est l'hypothèse majeure de ce travail, la frontière et la dualité qu'elle engendre offrent une clé de lecture fondamentale pour la lecture de l'espace urbain strasbourgeois. En nous inspirant du travail de Laurent Grison, nous pouvons dire que la frontière fonctionne dans cette ville comme une figure géographique fondatrice (Grison 2002) :

13 - Figure géographique car la frontière renvoie à « un modèle et une forme fondamentale de l'espace ", tout en déterminant le sens des représentations qu'elle porte. Cette figure géographique permet ainsi d'allier intimement la forme et le sens, quel que soit l'échelle de référence.

14 - Fondatrice en ce que la dualité franco-allemande sur laquelle l'espace urbain repose, permet de dévoiler sa structure profonde pour penser la ville dans sa composition matérielle, mais étroitement associée à l'imaginaire de l'identité qui la porte.

15 Une telle analyse permet dès lors d'approfondir l'approche frontalière de Joël Kotek, en permettant de penser, à différentes échelles spatiales et temporelles, l'évolution de la structure de Strasbourg, et ce autant sur le plan de son dessin que par les significations politiques successives qui lui ont été accordées.

\section{Les réinterprétations de la forme urbaine strasbourgeoise à la lumière du dualisme franco- allemand}

\section{A. Les centres symboliques, emblèmes des projets urbains successifs}

Le caractère partagé de Strasbourg, entre deux cultures, est lisible de manière diffuse à travers de nombreux détails inscrits dans l'architecture, la toponymie, certains modes de gestion municipale ou les pratiques de ses habitants. Ces éléments, bien qu'ils s'atténuent, font à la fois le charme et l'étrangeté de Strasbourg pour les visiteurs de passage. On peut distinguer dans la ville différents espaces où cette dualité est 
particulièrement vive, parce qu'ils correspondent à des lieux de condensation symboliques majeurs. Ils sont d'autant plus intéressants et centraux qu'ils ont été conçus de manière programmatique, comme des éléments pivots de projets régulateurs pour une interprétation globale de la ville.

Il faut rappeler qu'avant même de s'exprimer dans la composition urbaine, l'imposition politique du pouvoir royal, s'est affirmée dans l'espace religieux strasbourgeois. Avec le Rattachement de 1681, la monarchie française exigeait le rétablissement du culte catholique dans la cathédrale, en même temps qu'elle lui attribuait de droit le chœur de certaines églises paroissiales qui aujourd'hui encore restent marquées dans leur architecture par cette partition politico-religieuse. Malgré leur puissance hautement symbolique, ces partitions restent spatialement circonscrites. A l'échelle de la ville entière, l'expression de la dualité va trouver une expression particulièrement affirmée dans trois lieux qui déclinent les enjeux successifs du face-à-face national francoallemand. Chacun à sa manière fixe les enjeux historiques d'une époque dont il traduit les luttes symboliques dans l'espace urbain. Chacun offre le projet d'une réinterprétation globalisante de la ville à différentes échelles territoriales, qui conduisent du monument à la structure urbaine: la place Kléber, la place de la République, le Jardin des Deux Rives.

Figure 1 : Localisation des places de pouvoir dans l'espace urbain strasbourgeois

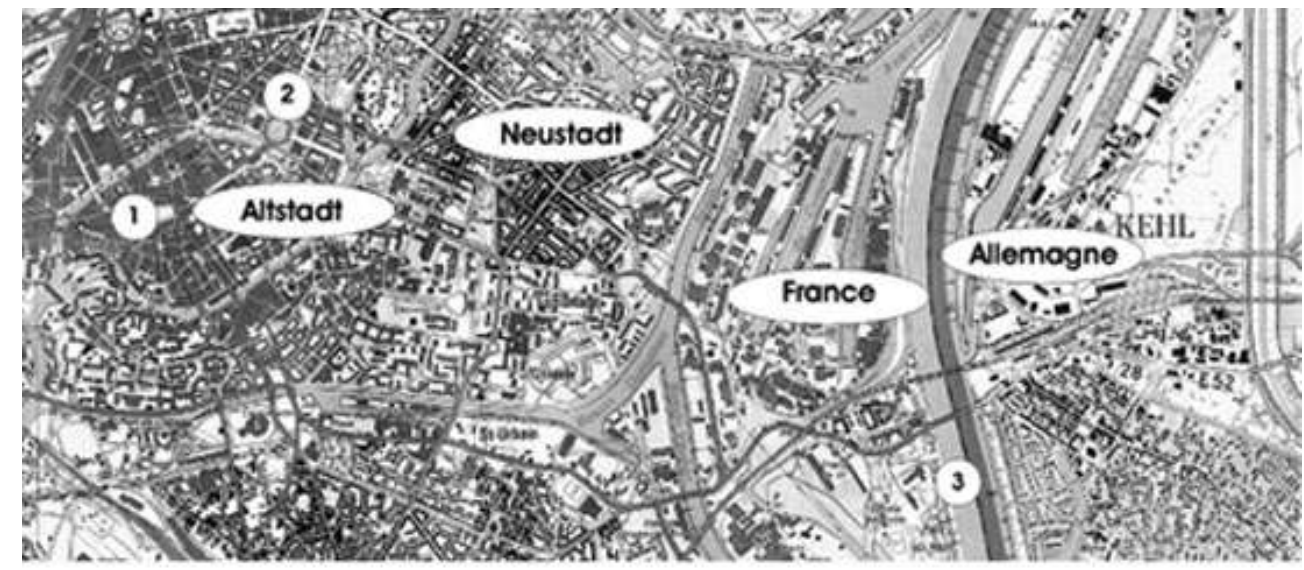

(1) Place Kléber

(2) Place de la République

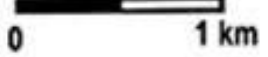

(3) Jardin des Deux rives

Altstadt : ellipse insulaire correspondant à l'hypercentre historique avec sa trame médiévale -

Neustadt : ou «ville allemande », quartiers correspondant à la trame haussmannienne mise en œuvre après l'annexion de 1870.

Extrait carte IGN 1/25 000 éd. 1999

\section{La place Kléber}

Le lieu qui constitue aujourd'hui le cœur de l'hypercentre strasbourgeois a été en partie dessiné sur un plan d'ensemble proposé en 1768 par l'architecte royal Jean-François Blondel, qui visait à en faire une place d'Armes. Il s'agissait bien pour le pouvoir français de marquer sa domination sur l'ancienne ville libre d'Empire par une grande composition « d'embellissement » et un système efficace de circulation militaire. 
Le principe de percée urbaine ne sera réalisé qu'un siècle et demi plus tard avec la
Grande Percée, selon un tracé quelque peu différent, puisqu'il s'agissait alors de
raccorder la place à la nouvelle gare centrale implantée à l'ouest. Sa réalisation
confirme alors la position centrale prise par la place Kléber dans l'espace
strasbourgeois. L'inauguration, en 1840, du monument dédié à Kléber (figure 2), fera
enfin de cet espace le symbole de l'appartenance de Strasbourg à la France. Aussi, après
l'annexion de 1870, c'est vers la statue du général révolutionnaire que convergeront
naturellement les manifestations francophiles.

Le principe de percée urbaine ne sera réalisé qu'un siècle et demi plus tard avec la
Grande Percée, selon un tracé quelque peu différent, puisqu'il s'agissait alors de
raccorder la place à la nouvelle gare centrale implantée à l'ouest. Sa réalisation
confirme alors la position centrale prise par la place Kléber dans l'espace
strasbourgeois. L'inauguration, en 1840, du monument dédié à Kléber (figure 2), fera
enfin de cet espace le symbole de l'appartenance de Strasbourg à la France. Aussi, après
l'annexion de 1870, c'est vers la statue du général révolutionnaire que convergeront
naturellement les manifestations francophiles.

Le principe de percée urbaine ne sera réalisé qu'un siècle et demi plus tard avec la
Grande Percée, selon un tracé quelque peu différent, puisqu'il s'agissait alors de
raccorder la place à la nouvelle gare centrale implantée à l'ouest. Sa réalisation
confirme alors la position centrale prise par la place Kléber dans l'espace
strasbourgeois. L'inauguration, en 1840, du monument dédié à Kléber (figure 2), fera
enfin de cet espace le symbole de l'appartenance de Strasbourg à la France. Aussi, après
l'annexion de 1870, c'est vers la statue du général révolutionnaire que convergeront
naturellement les manifestations francophiles.

Le principe de percée urbaine ne sera réalisé qu'un siècle et demi plus tard avec la
Grande Percée, selon un tracé quelque peu différent, puisqu'il s'agissait alors de
raccorder la place à la nouvelle gare centrale implantée à l'ouest. Sa réalisation
confirme alors la position centrale prise par la place Kléber dans l'espace
strasbourgeois. L'inauguration, en 1840, du monument dédié à Kléber (figure 2), fera
enfin de cet espace le symbole de l'appartenance de Strasbourg à la France. Aussi, après
l'annexion de 1870, c'est vers la statue du général révolutionnaire que convergeront
naturellement les manifestations francophiles.

Le principe de percée urbaine ne sera réalisé qu'un siècle et demi plus tard avec la
Grande Percée, selon un tracé quelque peu différent, puisqu'il s'agissait alors de
raccorder la place à la nouvelle gare centrale implantée à l'ouest. Sa réalisation
confirme alors la position centrale prise par la place Kléber dans l'espace
strasbourgeois. L'inauguration, en 1840, du monument dédié à Kléber (figure 2), fera
enfin de cet espace le symbole de l'appartenance de Strasbourg à la France. Aussi, après
l'annexion de 1870, c'est vers la statue du général révolutionnaire que convergeront
naturellement les manifestations francophiles.

Le principe de percée urbaine ne sera réalisé qu'un siècle et demi plus tard avec la
Grande Percée, selon un tracé quelque peu différent, puisqu'il s'agissait alors de
raccorder la place à la nouvelle gare centrale implantée à l'ouest. Sa réalisation
confirme alors la position centrale prise par la place Kléber dans l'espace
strasbourgeois. L'inauguration, en 1840, du monument dédié à Kléber (figure 2), fera
enfin de cet espace le symbole de l'appartenance de Strasbourg à la France. Aussi, après
l'annexion de 1870, c'est vers la statue du général révolutionnaire que convergeront
naturellement les manifestations francophiles.

Le principe de percée urbaine ne sera réalisé qu'un siècle et demi plus tard avec la
Grande Percée, selon un tracé quelque peu différent, puisqu'il s'agissait alors de
raccorder la place à la nouvelle gare centrale implantée à l'ouest. Sa réalisation
confirme alors la position centrale prise par la place Kléber dans l'espace
strasbourgeois. L'inauguration, en 1840, du monument dédié à Kléber (figure 2), fera
enfin de cet espace le symbole de l'appartenance de Strasbourg à la France. Aussi, après
l'annexion de 1870, c'est vers la statue du général révolutionnaire que convergeront
naturellement les manifestations francophiles. Gutenberg, alors cœur de l'ensemble urbain. Il aurait dû alors être marqué par une statue du monarque, placée devant l'hôtel de ville reconstruit (Sénat) et face à la cathédrale rendue au culte catholique depuis le rattachement de la ville à la France (1681).

Desservie par une large artère, la Place Royale ainsi aménagée était prévue pour accueillir les marchés, soulignant bien à ce propos «la concentration d'éléments symboliques autour de la statue en pied du Souverain : Abondance (marché), Justice (hôtel de Ville) et Religion (cathédrale) » (Garms 1978). Cette composition devait par là même oblitérer les symboles monumentaux de l'ancienne République municipale déchue (la Pfalz - palais de la municipalité -, le Neubau abritant l'administration municipale - la chancellerie et l'Hôtel de la Monnaie) auxquels elle se substituait. Plusieurs bâtiments ont effectivement été supprimés à cette époque et aujourd'hui seul subsiste le Neubau, siège de la Chambre de Commerce et d'Industrie.

La réalisation partielle de ce programme urbain marque de fait une translation de l'ancien centre de gravité politique de la ville, au profit d'un second point fort de la composition: la Place d'Armes. Du programme initial n'a été réalisé que le bâtiment militaire de l'Aubette, qui domine la place. Il lui donne aujourd'hui encore une tonalité classique qui n'est respectée sur aucun des autres côtés. Le vaste espace dégagé par la démolition du couvent des Franciscains au $16^{\mathrm{e}}$ siècle devient alors l'expression de la présence militaire française et du nouveau pouvoir royal. Nord-Ouest, « du côté de Paris » jusqu'à la porte des Bouchers au Sud, « qui conduit à l'Allemagne » (1). Le nouvel axe éventrant l'ancien tissu urbain avait pour projet l'alignement des façades classiques afin d'offrir aux étrangers arrivant en France une image à la hauteur de sa puissance (2). Mais la réalisation du projet tourna court devant l'hostilité des habitants et par manque de moyens financiers de la ville.

Revue Géographique de l'Est, vol. 47 / 2 | 2007 
Figure 2 : La place Kléber

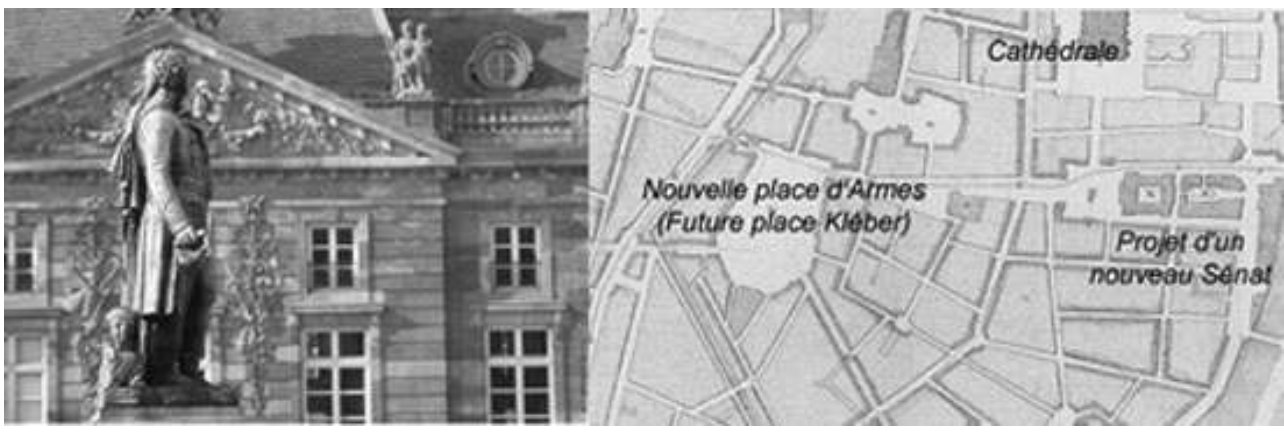

Statue du général Kléber et Aubette (a) - Projet non réalisé de Blondel pour Strasbourg 1773 (b)

Sources: Atlas historique des villes de France

\section{La place de la République}

l'ancienne place de l'empereur symbolise un deuxième espace fort de confrontation nationale. L'espace vert qui en constitue le cœur dessine une forme circulaire qui renvoie explicitement au globe impérial, insigne par excellence du pouvoir temporel. Il avait d'ailleurs été conçu pour accueillir en son centre la statue équestre de Guillaume $\mathrm{I}^{\mathrm{er}}$ de Hohenzollern. La place va progressivement se dessiner selon un vaste programme monumental: palais impérial (actuel palais du Rhin), bibliothèque impériale et régionale (actuelle Bibliothèque Nationale Universitaire), diète régionale (Landtag) qui abrite aujourd'hui le TNS, et deux bâtiments administratifs symétriques (l'actuelle Préfecture).

La jonction s'établit entre les deux villes par la place Broglie, qui sur le plan morphologique et fonctionnel s'apparente à un cours, terme toutefois inusité dans des contrées aussi septentrionales. Il fonctionnait comme le centre civique de la ville française post-révolutionnaire. Diverses institutions étaient abritées dans des hôtels particuliers classiques (services municipaux, préfecture, intendance, gouvernement militaire). L'ensemble est complété par l'opéra, construit dans la première moitié du $19^{\mathrm{e}}$ siècle pour achever la perspective de la promenade.

En le dédoublant, le projet allemand cherche à déplacer les fonctions centrales vers ce qui est appelé à constituer le nouveau centre de la représentation politique: les ministères, la diète régionale et le palais de l'empereur. Et ceci avec d'autant plus de force que la place impériale organise la composition des nouveaux quartiers selon un axe de prestige qui lie le nouveau centre politique au nouveau pôle spirituel (Palais Universitaire), débutant au palais impérial jusqu'au palais universitaire à l'Est. Les tracés des grands axes assurent un lien visuel fort avec la flèche de la cathédrale, pour bien souligner la filiation des nouveaux espaces avec l'héritage insigne de la ville libre du Saint-Empire.

Toute une symbolique urbaine se développe alors sur la mise en perspective systématique de l'intégration de Strasbourg à l'Ancien et au Nouvel Empire. L'extension est conçue pour accueillir une population allemande et trouve sa place dans une politique de germanisation de l'Alsace et d'expansion urbaine. A travers ces réalisations urbaines de prestige, l'objectif politique affiché est bien alors de promouvoir un nouvel ordre, d'impressionner une population autochtone par les bienfaits de la nouvelle 
administration. La vocation de Strasbourg était alors de devenir la capitale du Reichsland, une vitrine de la puissance matérielle et intellectuelle du nouvel empire, largement ouverte sur le Rhin avec ses aménagements portuaires. Ce n'est que très récemment avec les aménagements du tramway et une fonction de correspondance que la place réaménagée est rentrée quelque peu en grâce auprès des Strasbourgeois.

Figure 3 : Place de la République

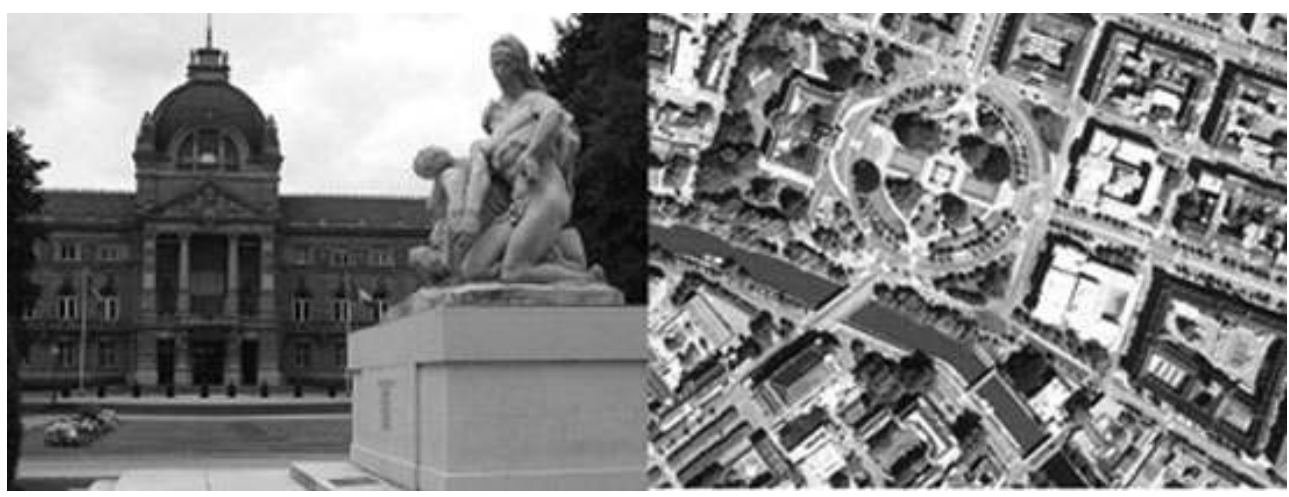

Monument aux Morts et Palais du Rhin (a) - Photo aérienne prise entre la Neustadt allemande et la ville ancienne (b)

Sources : cliché personnel et CUS

\section{Le Jardin des Deux Rives}

Le troisième élément fort de cette rhétorique urbaine franco-allemande a été réalisé en 2004. Il marque dans l'espace urbain un retournement de la signification de la frontière et du rapport entre les deux pays. Cet aménagement paysager transfrontalier occupe plus d'une centaine d'hectares de part et d'autre du Rhin et se présente comme un signal fort de la réconciliation entre les deux pays : le symbole de son ancrage européen (figure 4).

Figure 4 : Le Jardin des Deux Rives

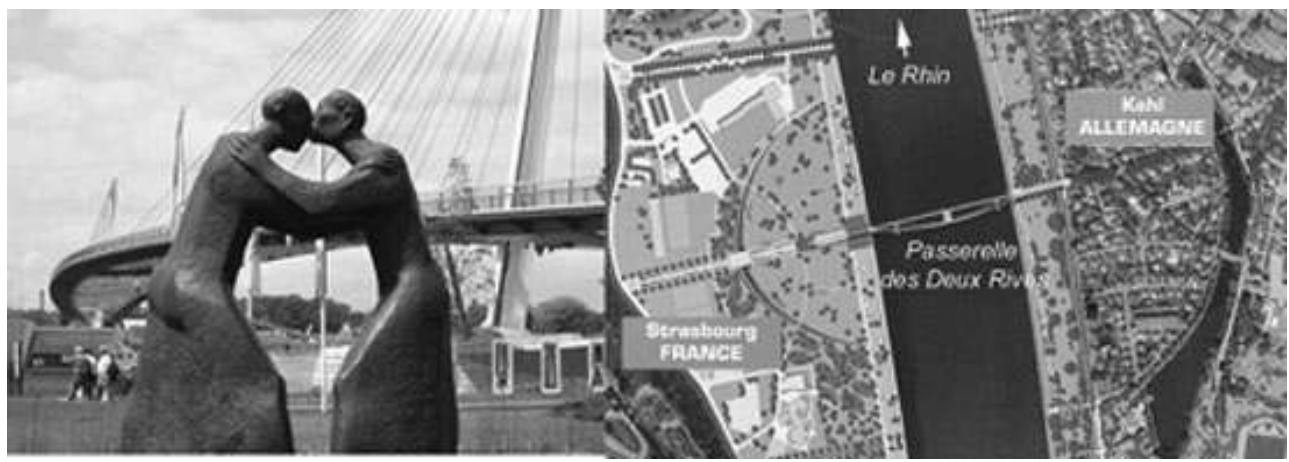

«Die Begegnung - la rencontre » en arrière-plan la passerelle des Deux Rives (a) - Plan du Jardin traversé par le Rhin et la frontière (b)

Sources : cliché personnel et CU

29 Sa réalisation a permis de requalifier d'importantes friches douanières, militaires et industrielles, ouvrant un programme urbain de grande ampleur tant du côté français (Front de Neudorf - Porte de France - Quartier du Danube) que du côté kehlois. Cet espace apparaît comme un puissant marqueur du changement sémantique de la 
frontière, symbolisé dans sa structure même par la très belle passerelle réalisée par l'architecte parisien Marc Mimram, où deux cheminements convergent jusqu'à une plate forme-belvédère qui surplombe le Rhin au milieu de son chenal, à l'aplomb de la ligne de partage entre les deux pays.

Le Jardin des Deux Rives apparaît aussi comme l'élément central d'une large composition urbaine en cours de réalisation. Il ouvre vers l'Allemagne un axe structurant qui, partant de la place de l'Etoile à Strasbourg, vient suturer les anciennes zones non aedificandi des fortifications déclassées, en bordure méridionale immédiate de l'hypercentre (les Fronts de Neudord cf. figure 1). Le long de ce nouvel axe, de nombreux équipements publics et des programmes de logement sont planifiés, et en partie déjà réalisés.

31 La centralité du Jardin et des espaces adjacents apparaît à l'échelle de l'agglomération transfrontalière. Encore peu exploitée aujourd'hui, elle est certainement appelée à jouer un rôle pivot, bien desservi par les transports frontaliers (route et rail) et surtout parce qu'il peut être désormais perçu comme un espace politiquement commun aux habitants des deux rives. Toutefois, peu d'éléments de centralité s'y sont fixés alors que les fonctions traditionnelles liées à la coupure frontalière (hôtellerie, banque, douane) ont disparu. Le retournement ne peut être effectif sans une volonté politique d'affirmation urbain qui peine à trouver forme côté français, malgré les nombreux les projets. Ce n'est qu'avec leur mise en chantier que le point frontalier, structurellement en marge de l'espace urbain, pourra occuper une réelle fonction d'articulation dans la redéfinition d'une aire urbaine transfrontalière.

Les trois lieux évoqués scandent des moments déterminants de l'histoire urbaine. A leur façon, ils se font les interprètes des rapports franco-allemands, en ponctuant l'espace urbain. On voit bien comment le motif de partage traverse la ville. Il en assure une lisibilité qui s'inscrit dans un programme symbolique que l'on peut décliner à diverses échelles spatiales ( $\mathrm{du}$ monument à la composition urbaine) et temporelles (selon un travail renouvelé de cette mémoire sur l'appartenance nationale). Mis en perspective, les trois lieux indiquent aussi un certain nombre de ruptures fortes : celle de l'échelle qui vient appuyer un plan régulateur, mais aussi celles de la signification des lieux, qui mérite également d'être analysée.

\section{B. Surimpositions et subversion : une réinterprétation dialectique des lieux de contact}

33 La double inscription de Strasbourg dans l'histoire française et allemande a laissé bien des marques. Ainsi chacun des lieux de centralité évoqués précédemment est traversé par une confrontation symbolique qui en structure la portée. La dualité peut se définir comme ce qui est double, c'est-à-dire qui est répété, comme à ce qui a deux aspects, souvent opposés d'ailleurs. Le développement qui suit va montrer comment ces lieux de centralité sont travaillés par une confrontation symbolique qui en construit la dualité interne. Comment chacun de ces lieux est traversé par une tension symbolique propre qui conduit au renversement et à la subversion des valeurs dont ils sont les porteurs.

Parmi les trois espaces retenus, la place Kléber est sans doute le lieu où la démonstration de retournement symbolique est la moins patente dans la pierre. Elle a surtout été marquée par des luttes symboliques dont les traces sont aujourd'hui peu visibles. La statue et les cendres du général Kléber, qui avaient été transférées durant 
l'occupation nazie sont rétablies en 1946. La place elle-même avait été débaptisée par l'occupant pour devenir la Karl-Ross-Platz, du nom de l'autonomiste alsacien exécuté en 1940 par l'armée française. Le bâtiment de l'Aubette porte les stigmates des conflits : incendié par le bombardement du siège allemand de 1870, il a été reconstruit pour être transformé en conservatoire de musique. Le style martial du bâtiment français original a été atténué par l'administration prussienne avec l'ajout d'une toiture mansardée et de médaillons floraux, encadrant les bustes de compositeurs en hautrelief qui ornent toujours la façade.

Une réhabilitation est programmée, qui va le rendre solidaire d'un bâtiment néorenaissance, typique de l'historicisme allemand du début du siècle passé ! Si l'on se réfère au Guide Gallimard, le décor hétéroclite de la place " laisse apparaître un côté français inachevé (l'Aubette), un côté allemand fait de plusieurs bâtiments de style wilhelmien, et, au fond de la place, un côté alsacien que constituent quelques maisons à colombage » (Gallimard, 1996). A quoi il faut ajouter les éléments plus modernes : la Tour Valentin-Sorg qui domine l'horizon au nord-ouest, élément de la Reconstruction consécutive aux bombardements de 1944 et la Maison Rouge venue remplacer dans les années 1970 un bâtiment de facture plus classique.

Pour la place de la République, le renversement des perspectives symboliques est plus net avec le changement de pouvoir. Dès le 9 novembre 1918, la statue équestre de Guillaume $\mathrm{I}^{\mathrm{er}}$ est mise à bas dans une ambiance insurrectionnelle. Elle sera remplacée en 1936 par un monument aux morts qui représente une mère éplorée tenant dans ses bras ses deux fils, l'un tombé pour la France, l'autre pour l'Allemagne (figure 3). Au cœur de l'urbanisme triomphant du militarisme prussien se trouve désormais une figure de recueillement et de deuil. Comme pour souligner cette double identité, une fontaine conçue par l'artiste Tomi Ungerer est inaugurée en 1988 à la jonction entre la ville ancienne et la Neustadt. Si les deux arches en brique rappellent le bi-millénaire de la fondation romaine de la ville, la pile centrale prend appui sur une tête de Janus, dont le visage est tourné vers les deux parties de la ville. Elle fonctionne comme un trait d'union paradoxal, symbolisant à la fois l'ambiguïté du passage et, peut-être à sa manière, la double culture.

Bien que transformé par une action paysagère de grande ampleur, célébrant la réconciliation franco-allemande, l'espace du Jardin des Deux Rives demeure fortement imprégné par la présence de nombreux monuments aux morts et de plaques commémoratives d'exécutions sommaires perpétrées durant la période nazie. Ceux-ci sont tournés vers la frontière comme autant de signes de révolte et de défi vers le pays des anciens bourreaux. Les bunkers désarmés et les diverses stèles sont aujourd'hui inclus dans l'enceinte du Jardin et deviennent autant de souvenirs d'une mémoire douloureuse dans l'espace de la réconciliation d'aujourd'hui.

On peut tenter de résumer succinctement l'évolution symbolique des sites à travers le tableau ci-dessous.

En systématisant cette première lecture, on pourrait même tenter une interprétation structurale (Desmarais, 2003) de l'espace urbain strasbourgeois. A partir d'une succession d'oppositions binaires et nationales, le lieu politiquement investi par le pouvoir émergent porte le projet d'une nouvelle centralité urbaine, politique et géographique (Tableau 1). Celle-ci d'ailleurs accompagne souvent la croissance de la ville par une translation successive. Aussi est-ce toujours à partir de cette centralité que le pouvoir va chercher à ordonner et interpréter idéologiquement l'ensemble de 
l'espace urbain. Si l'on suit cette lecture, on peut décrire le rôle central des places dans les redéfinitions successives du centre politique de la ville (figure 5).

Tableau 1 : Evolution fonctionnelle et symbolique des trois places strasbourgeoises

\begin{tabular}{|c|c|c|c|c|}
\hline Lieux emblématiques & Domination symbolique & Fonction initiale & Monument(s) actuel(s) & $\begin{array}{c}\text { Centralité à l'échelle } \\
\text { de la composition } \\
\text { urbaine }\end{array}$ \\
\hline Place Kléber & France > Allemagne & Place dAArmes & Statue du Général Kéber & Ellipse insulaire \\
\hline Place de la République & Alemagne > France & $\begin{array}{c}\text { Centre politico- } \\
\text { administratif }\end{array}$ & $\begin{array}{c}\text { Monument aux morts } \\
\text { (Alt- et Neustact) }\end{array}$ & Ville étendue \\
\hline Jarcin des Deux Rives & France = Allemagne & $\begin{array}{c}\text { Lieux de commémoration } \\
2^{e} \text { GM Espaces militaires } \\
\text { et douaniers } \\
\text { et réconciliation } \\
\text { - passerelle } \\
\text { - statue rive allemande }\end{array}$ & $\begin{array}{c}\text { Aggomération } \\
\text { transfrontalière }\end{array}$ \\
\hline
\end{tabular}

La place Gutenberg procède elle-même de la même logique, puisqu'en accueillant le centre civique de la République urbaine, elle signifie, au $13^{\mathrm{e}}$ siècle, l'affirmation des bourgeois de Strasbourg face au pouvoir épiscopal/parvis de la cathédrale. La place d'Armes (place Kléber) marque la domination du pouvoir monarchique français sur la cité (après 1681), de même que la réalisation de l'actuelle place de la République (après 1871) fait émerger une nouvelle centralité du pouvoir à la charnière entre les nouveaux quartiers (Neustadt) et l'ancienne ville (Altsdtadt). Dans cette logique, le Jardin des Deux Rives et ses abords occupent une situation potentiellement similaire, puisqu'il se place au cœur de l'Eurodistrict qui regroupe la Communauté urbaine de Strasbourg et l'arrondissement allemand voisin de l'Ortenau.

Figure 5 : Les places dans la définition de la centralité politique et urbaine de Strasbourg

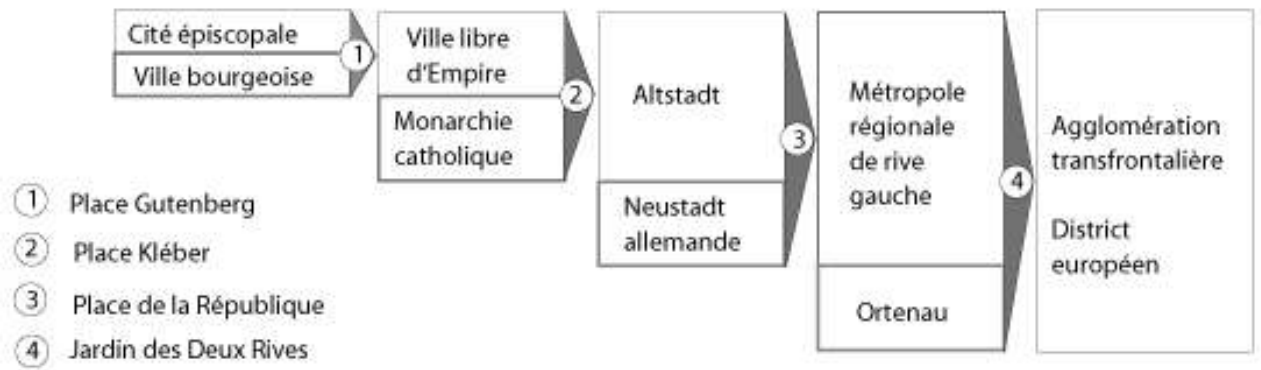

41 Si de nombreuses villes européennes partagent avec Strasbourg la succession des appartenances nationales, la singularité de cette dernière réside indéniablement dans le nombre de changements de souveraineté et plus encore dans l'importance symbolique que ces changements revêtent dans la structure urbaine. Il ne s'agit pas seulement d'une simple succession chronologique. Leurs coexistences dans l'espace actuel de la ville sont autant de repères signifiants dans la structure urbaine. Les places représentent alors comme des pivots territoriaux, chargés de significations cumulées. Elles portent à travers l'histoire un travail continu d'interprétation politique qui illustre la dualité nationale, et scandent l'évolution même du sens de la frontière pour la ville.

Toutefois, la lecture urbaine proposée ne relève pas de la seule lecture historique. Elle doit aussi suggérer une interprétation plus dynamique de la position strasbourgeoise, pour laquelle la perspective franco-allemande peut être véritablement un levier mobilisateur de son projet. Dans cette perspective, la mise en cohérence en termes de 
planification stratégique urbaine est pourtant loin d'être réalisée, voire même pleinement acceptée tant par la population que par les élus.

\section{Dividendes et ambiguïtés strasbourgeoises autour de la symbolique franco-allemande}

\section{A. Strasbourg, géo-symbole du couple franco-allemand}

Cette fonction de lieu symbolique de rencontre entre les deux nations, Strasbourg l'incarne tout particulièrement. Plus qu'Aix-la-Chapelle, Reims, Verdun, Versailles (Morizel J., Möller Horst 1995) ou plus récemment encore les plages de Normandie, la ville est un haut-lieu de mémoire commune, d'autant plus puissant qu'il permet d'offrir l'image d'un passé moins fortement tragique.

Pour les Français et les Allemands, c'est bien à Strasbourg que les deux pays se touchent, géographiquement, par le Rhin. Cette réalité n'appartient pas qu'au passé, elle est certainement un enjeu prospectif majeur pour la ville. Pourtant Strasbourg éprouve une difficulté certaine à reconnaître et à tirer parti de sa situation de proximité entre France et Allemagne, peut-être ne veut-elle pas s'y laisser enfermer. Drapée dans son rôle de capitale parlementaire européenne, elle n'a jamais été localement un pôle de coopération fort dans la région du Rhin supérieur, où les modèles sont à chercher vers Bâle (Régio TriRhena) ou Karlsruhe (Pamina). Localement, la dynamique du rapprochement franco-allemand semble peu prononcée en dépit des encouragements et des attentes au plus haut niveau.

Il est vrai que Strasbourg s'est longtemps appliquée à atténuer les traces de son passé allemand le plus récent, s'appuyant plus sur son histoire française ou le prestigieux souvenir de son rôle de centre commercial et culturel rhénan, à une époque où elle était encore ville libre d'Empire, c'est-à-dire à une époque antérieure aux conflits entre nationalités. Après 1918 d'abord, et surtout après 1945, se construit un oubli politique de la proximité de l'Allemagne, alors même que la ville se forge un destin européen pour dépasser les confrontations nationales qui l'ont tant marquée.

L'espace frontalier accueille essentiellement des activités industrielles lourdes rejetées dans la zone portuaire, le long de la frontière, et qui voit se développer un glacis d'un nouveau type. Contrairement aux métropoles voisines de Bâle ou de Karlsruhe qui ont su nouer d'étroites relations transfrontalières de proximité, Strasbourg s'ouvre tardivement à son voisin immédiat. L'affermissement des liens entre la France et l'Allemagne au sein de l'Union Européenne vient compenser son faible empressement à la coopération transfrontalière.

Avec la ville moins prestigieuse de Sarrebruck qui abrite l'administration de l'Université franco-allemande, elle représente de fait le seul pôle frontalier de prestige entre les deux pays, susceptible d'accueillir des fonctions supérieures. Aussi les gouvernements successifs des deux pays trouvent-ils dans la ville un lieu évident d'implantation de structures communes, que vient renforcer son statut européen. Encore une fois, l'enjeu structurant semble être guidé par des considérations d'ordre politico-symboliques. La dynamique demeure largement impulsée par les Etats, comme si la ville se limitait à un rôle de gestionnaire largement passif de cette perspective. 
Ainsi l'Eurocorps, l'OFAJ (Office franco-allemand pour la Jeunesse) ou la chaîne de télévision culturelle Arte, qui en sont les exemples les plus marquants, ont trouvé à Strasbourg un lieu qui convenait à leur projet bi-national et européen. Certaines entreprises privées ont pu s'appuyer sur cette même rhétorique. C'est le cas du groupe Aventis issu du rapprochement dans la chimie de l'allemand Hoechst et du français Rhône Poulenc, lorsqu'il décida d'établir son nouveau siège social dans l'aire métropolitaine strasbourgeoise. Son implantation dans le parc d'activités de 3E (Espace Economique Européen) à Schiltigheim n'aura été qu'éphémère, puisque la fusion du groupe avec Sanofi marque le retour de la direction à Paris, en 2004.

L'attrait de la ville se vérifie aussi pour de nombreux couples franco-allemands venus s'établir dans la région urbaine pour profiter de l'accessibilité bi-nationale qu'offre la région strasbourgeoise pour la recherche d'un emploi ou la scolarisation des enfants. Sur le moyen terme, le développement de telles initiatives marque un enjeu qui est déterminant pour la ville et son image. Comme pour Arte et l'Eurocorps, les structures embryonnaires pourraient bien être appelées à s'élargir à d'autres pays de l'Union et avoir des retombées positives grandissantes. On peut souligner qu'une des questions cruciales pour son destin européen, à savoir le maintien du siège du Parlement, dépend de la qualité de l'accessibilité de la ville. Il est indéniable que, là encore, le destin de la ville se joue entre la France et l'Allemagne.

L'échec relatif du désenclavement aérien de Strasbourg tient en partie au fait de n'avoir pu trouver localement les conditions d'une entente transfrontalière. Un certain nombre de lignes aériennes ne sont maintenues qu'au titre de leur fonction européenne, étant pour cela largement subventionnées par l'Etat français. Même si les données d'un aéroport bi-national, comme il existe à Bâle, ne sont pas simplement reproductibles, il serait souhaitable de mettre en cohérence les offres aériennes régionales. Malgré les difficultés d'exploitation liées à l'éloignement, une infrastructure aéroportuaire partagée avec Karlsruhe avait été envisagée à deux reprises. D’ailleurs, l'aéroport de Baden-Baden occupe en partie ce rôle avec l'accueil de la compagnie lowcost Ryanair initialement basée à Strasbourg-Entzheim (Arbaret-Schultz C., Beyer A., 2003).

\section{B. Franco-allemande ou/et européenne, les ambiguïtés strasbourgeoises}

51 L'ancrage franco-allemand est sans doute pour Strasbourg le seul moyen d'exister dans une logique de métropoles européennes, notamment face à Bruxelles. Elle offre incontestablement à l'agglomération une meilleure visibilité et lui assure une masse démographique critique de près d'un million d'habitants. Trop insister sur le rôle franco-allemand de la ville n'est pas sans risque de nuire à l'image d'une capitale européenne, car Strasbourg court alors le danger d'être simplement perçue comme symboliquement confisquée par deux poids lourds de la construction de l'Union. Toutefois, les deux logiques se complètent plus qu'elles ne s'opposent. Ainsi, la projection de Strasbourg sur le Rhin avec le Jardin des Deux Rives et ses développements urbains ont été conçus comme le gage incontestable de l'ouverture européenne de la ville au-delà de la frontière nationale. Le projet urbain transfrontalier devait lui-même être le support d'une Europe vécue dans la proximité et la citoyenneté 
quotidienne, à l'opposé d'une construction européenne technocratique et abstraite, que les élus strasbourgeois aiment à associer à la capitale belge.

Dans cette perspective, la création de l'Eurodistrict Strasbourg-Ortenau est particulièrement instructive des ambiguïtés strasbourgeoises. Lancé le 22 janvier 2003 par les chefs d'Etats français et allemand pour marquer le $40^{\mathrm{e}}$ anniversaire du traité de l'Elysée, le projet se présente comme la volonté, au sommet de l'Etat, d'approfondir les processus locaux d'intégration européenne. Dans la représentation des dirigeants nationaux, il ne fait aucun doute que l'agglomération strasbourgeoise est le lieu idéal pour structurer ces nouvelles proximités et assumer un rôle de pilote pour d'autres régions frontalières. On note, une fois encore, que la dynamique de contact est initiée par l'action des Etats et sous-tendue par la portée symbolique du rapprochement franco-allemand. Pourtant, malgré un parrainage prestigieux et des encouragements réitérés, le projet a peiné auprès des élus locaux à qui en était confié la réalisation. L'Eurodisctrict ne sera péniblement mis en place que deux ans plus tard, sur des bases minimales assez loin de l'ambition initiale. Cette situation souligne le décalage entre les projections symboliques des cabinets ministériels, les stratégies de terrain des élus locaux et la perception de la population, à la fois lassée par des discours grandiloquents peu suivis de faits, et finalement dans sa grande majorité, faiblement concernée par la proximité frontalière.

Sur le plan local, certains éléments ont indiscutablement contribué à freiner le rapprochement: au-delà des difficultés de relations personnelles entre les têtes de l'exécutif de couleurs politiques différentes, il faut rappeler les profils hétérogènes des partenaires, même si le principe de découpage avait retenu des territoires de poids démographique comparable, soit 450000 habitants de part et d'autre de la frontière. L'Ortenau se présente comme un territoire composé de villes moyennes et petites, politiquement morcelé et encore largement imprégné d'une mentalité rurale (son territoire est composé pour près de la moitié par les zones montagneuses de la Forêt Noire). Ce territoire considère avec une certaine appréhension le rapprochement avec une métropole strasbourgeoise dominée par un exécutif puissant et souvent peu enclin au compromis. De leur côté, les élus alsaciens ont sans doute craint de devoir partager avec leurs voisins allemands le statut européen (pourtant fragile) de Strasbourg, et avec lui ses dividendes. Dans sa forme actuelle, l'Eurodistrict reste ainsi une amorce qui exprime encore la crainte et l'hésitation devant la perspective d'un avenir commun et d'éventuels engagements institutionnels et financiers. Derrière les communiqués laudateurs sur l'avancement de la coopération transfrontalière, la presse régionale se fait aussi l'écho des commerçants strasbourgeois qui doivent se mesurer à l'attractivité des commerces d'Outre-Rhin, notamment à Kehl. A l'inverse, avec l'évolution du marché immobilier français, les Badois voient affluer chez eux des Strasbourgeois de plus en plus nombreux, en quête d'offres bien meilleures marchées. Si elle n'est pas sans créer de nouvelles tensions à court terme, cette situation inédite porte aussi des perspectives d'intégration et d'échanges plus poussés entre les deux rives du Rhin. En somme, la construction effective du territoire transfrontalier suit un rythme propre qui demandera sans doute plus de temps que n'avait pu l'envisager l'action symbolique du pouvoir politique. Elle est à l'image des ambitieux desseins urbains projetés autour du Jardin des Deux Rives, où les réalisations de l'interface urbaine et frontalière sont encore à l'état de projet. 


\section{Conclusion}

S'il semble engagé, le projet urbain de construction d'une agglomération francoallemande exige encore un travail important de la part des acteurs strasbourgeois, pour se réapproprier un passé jusqu'alors subi et largement occulté dans une lecture nationale. Faut-il rappeler que Strasbourg doit en partie sa fonction européenne au fait qu'elle incarnait particulièrement les déchirements du continent?

L'affirmation d'une perspective d'articulation transnationale ouvre assurément à la ville d'intéressantes stratégies de positionnements fonctionnels et symboliques sur le long terme. Dans ce sens, le modèle est bien à chercher dans le $18^{\mathrm{e}}$ siècle, avant le raidissement des nationalismes, alors que la ville remplissait pleinement un rôle de pont économique et culturel entre les espaces germaniques et français.

La valorisation sur le plan urbain de la dualité apparaît comme un principe remarquable et pourtant curieusement méconnu dans la construction physique et sémantique de la ville. Mise en perspective, cette dualité enfin assumée permet alors le dialogue entre les espaces et les échelles urbaines qui sont ainsi étroitement articulées dans le temps et l'espace franco-allemand. Par comparaison, il est notable que les fonctions européennes qui y sont implantées depuis un demi-siècle n'ont jamais servi d'éléments véritablement structurants à une composition urbaine de grande ampleur.

Revendiquer et construire un statut de ville partagée entre les deux pays n'est toutefois pas sans risque, comme le rappelle la difficile mise en œuvre de l'Eurodistrict Strasbourg-Ortenau. Il s'agit d'affirmer une nouvelle identité urbaine qui ne soit pas fondée sur l'assimilation nationale (comme cela a pu être le cas dans le passé), mais sur l'articulation explicite des identités nationales. Cela suppose alors de traduire le dessin urbain en véritable projet de positionnement politique.

\section{BIBLIOGRAPHIE}

ARBARET-SCHULTZ C., BEYER A. (2003). - « Polycentrisme aéroportuaire et polycentrisme urbain sur les frontières nord-orientales de la France ", in Le polycentrisme, un projet pour l'Europe, PUR, Rennes, pp. 127-139.

BEYER A., WULFHORST G. (2005). - « L'interconnexion ICE/TGV à Strasbourg, l'utopie d'une croisée de la grande vitesse franco-allemande ", $5^{\mathrm{e}}$ Rencontre de Mâcon « Réseaux en question : utopies, pratiques et prospective », 30 juin- $1^{\text {er }}$ juillet 2005.

CALAY V. (2003). - « La production imaginaire d'une capitale de l'Europe : vers la territorialisation d'une identité collective européenne? », Belgeo, No. 4, 2003.

CLAVAL P. - « Les interprétations fonctionnalistes et les interprétations symboliques de la ville », Cybergéo No. 81.

COLLECTIF (2000). - Strasbourg 1900 : naissance d'une capitale. Colloque, Strasbourg, $1^{\mathrm{er}}-4$ décembre 1999, Paris, Somogy. 
COLLECTIF (1996). - Strasbourg, urbanisme et architecture des origines à nos jours, Oberlin, Strasbourg, $297 \mathrm{p}$.

CORNELISSEN C., FISCH S., MAAS A. (1997). - Grenzstadt Strassburg - Stadtplanung, kommunale Baupolitik und Öffentlichkeit 1870-1940, Stuttgart, Röhrig-Universitätsverlag.

DEBARBIEUX B. (1996). — « Le lieu fragment et symbole du territoire », in Espaces et sociétés, No.

82-83.

DESMARAIS G., RITCHOT G. (2001). - La géographie structurale, Paris, L'Harmattan.

GARMS Jörg (1978). - Le plan d'urbanisme de Strasbourg dressé par Jacques-François Blondel en 1764-1769 ", in Cahiers Alsaciens d'Archéologie, d'Art et d'Histoire, tome XXI, pp. 103-141.

GRISON L. (2002). - Figures fertiles. Essai sur les figures géographiques dans l'art occidental, Nîmes, Editions Jacqueline Chambon, Collection Rayon Art, 148 p.

GUIDES GALLIMARD (1996), Alsace, 165 p.

котек J. (1996). - L'Europe et ses villes frontières, Bruxelles, Editions Complexe, 329 p.

MONNET J. (1998). - « La symbolique des lieux : pour une géographie des relations entre espace, pouvoir et identité », Cybergéo, No. 56.

MONNET J. (1993). - La ville et son double. La parabole de Mexico. Essais et recherches, Paris, Nathan.

MORIZEL J., MÖLLER H., Dir. (1995). - Allemagne France. Lieux et mémoire d'une histoire commune, Paris, Albin Michel, Histoire, $240 \mathrm{p}$.

NOHLEN K. (2003). - « Strasbourg après 1871 : réorganisation des espaces », in Turrel D. (Dir.), Villes rattachées, villes reconfigurées $16^{e}-20^{e}$ siècle, Tours, Presses Universitaires François Rabelais.

NOHLEN K. (1997). - Construire une capitale, Strasbourg impérial de 1870 à 1918 : les bâtiments officiels de la place impériale, Publications de la Société Savantes d'Alsace, tome 56.

PETITDEMANGe G. (2002). - « La mémoire de la guerre dans une ville frontière, Strasbourg », pp. 33-53 in Raphaël F. (et al.), Mémoire de pierre, mémoire de papier, Strasbourg, Presses Universitaires de Strasbourg.

PINOL J.-L., Dir. (1996). - Atlas historique de France, Paris, Hachette, dont le chapitre consacré àStrasbourg pp. 121-143.

REITEl B., ZANDER P., PIERMAY J.-L., RENARD J.-P., Dir. (2002). - Villes et frontières. Paris, Anthropos (Coll. « Villes »), 275 p.

\section{NOTES}

Où l'auteur limite la symbolique européenne aux seuls paysages des bâtiments institutionnels de l'Union (Calay 2003)

Ainsi les églises de « simultaneum » de St-Pierre le Vieux et Saint-Pierre le Jeune protestant, dont les bâtiments ont été partagés, obligeant les Protestants à ouvrir partiellement leurs églises au culte catholique.

Selon les indications portées le « projet idéal pour le centre de Strasbourg » de J.-F. Blondel in J.L. Pinol (1996) p. 132.

Le bâtiment édifié entre 1765 et 1778 était destiné à recevoir un corps de garde, la chambre des logements militaires et le local où se donnait à l'aube de chaque jour les ordres destinées à la 
garnison, d'où son nom « d'Aubette » in Strasbourg. Urbanisme et Architecture des origines à nos jours, p. 95.

Cette référence a été renforcée par une statuaire renvoyant à l'époque révolutionnaire (en hommage à La Marseillaise qui y aurait été composée et au général Kellermann). Elle est complétée par le monument au Général Leclerc, dont l'épopée n'est pas sans s'inspirer des Soldats de l'An Deux.

L'Eurocorps est un état major international capable de commander une force allant jusqu'à 60000 hommes. Créé en 1992 et déclaré opérationnel en 1995, il est une initiative franco-allemande à laquelle se sont successivement joints la Belgique, l'Espagne puis le Luxembourg.

\section{RÉSUMÉS}

Successivement ville-frontière, puis ville frontalière, Strasbourg est aujourd'hui en passe de se constituer en intercommunalité transfrontalière. Aux conflits d'une ville partagée, enjeu de pouvoirs politiques et militaires nationaux, a succédé une période à la fois de déni et de repli. L'évolution du sens de la frontière offre aujourd'hui à Strasbourg l'occasion d'un important redéploiement territorial et stratégique. Dans cette perspective, partant de l'interprétation de trois lieux emblématiques de la double appartenance strasbourgeoise (la place Kléber, la place de la République et le Jardin des Deux Rives), l'article montre comment ces espaces peuvent se présenter à la fois comme des éléments-clés de la structure de la ville et surtout permettre d'inscrire dans le temps long un projet global pour l'agglomération transfrontalière de demain.

Strasbourg has changed its geopolitical position through times. The disputed town on the frenchgerman borderland (ville-frontière) has become a french town situated near the german border (ville frontalière). A time of denial and retirement had followed the period when Strasbourg was submitted to political and military powers between the two nations. The contemporary evolution of the intra-european borders gives nowadays Strasbourg a real opportunity for a new development, as the town is gaining a crossborder identity. The paper tries to give an geographical interpretation of this evolution through the spatial organization of three public squares (place Kléber, place de la République and the Jardin des Deux Rives on the Rhine river). They may serve as key elements in the structure of the town history and future.

Zuerst als Grenzstadt und dann als Stadt an der Grenze, ist jetzt Strassburg dabei eine grenzübergreifende Metropolregion zu bilden. Nach den langwierigen Konflikten einer geteilten Stadt, Zankapfel politischer und militärischer Nationalmächte, war eine Zeit der Verweigerung und des Verschliessens eingedrungen. Die gegenwärtige Öffnung der Grenze bietet aber für Strassburg die Chance einer neuen Entwicklung. In dieser Hinsicht ist die Bedeutung drei emblematischer Orte, die auf die Doppelangehörigkeit von Strassburg deuten, zu achten (Kleberplatz, place de la République, der Garten der Zwei Ufer). Der Artikel betont in dieser Hinsicht wie diese Orte als unvermeidliche Schlüsselelemente der städtischen und symbolischen Struktur der Stadt die Zukunft mit einer endlich versöhnten Vergangenheit vereint. 
INDEX

Schlüsselwörter : deutsch-französich, Eurodistrikt, Grenze, grenzübergreifende Beziehungen, Stadtraum, Strassburg

Keywords : boundary, crossborder relations, Eurodistrict, french-german relation, Strasbourg, urban space

Mots-clés : espace urbain, Eurodistrict, franco-allemand, frontière, Strasbourg, transfrontalier

\section{AUTEUR}

ANTOINE BEYER

Université Paris 4 (Paris- Sorbonne) 191, rue Saint-Jacques 75005 Paris France -

antoinebeyer@yahoo.fr 